\title{
Absceso de herida operatoria por Scedosporium prolificans: Primer aislamiento en Chile. Revisión de la literatura
}

\author{
M. CRISTINA DÍAZ J., CHRYSTAL JULIET L., \\ ARACELI MONZÓN DE LA T. y JUAN LUIS RODRÍGUEZ -TUDELA
}

\section{Surgical site abscess by Scedosporium prolificans: first report in Chile and review}

The aim of this article is to report the first case of infection by Scedosporium prolificans in our country. The patient corresponds to a 27 year-old man with chronic renal failure, hypertension, submitted to a renal transplant, in treatment with cyclosporine, azathioprine and prednisone. Twenty five days after the transplant, he noticed a fluctuating increase of volume under the surgical wound. In three consecutive purulent exudates and in tissue obtained at surgery, Scedosporium prolificans was isolated as an only agent. The histopathological study revealed structures compatible with septate hyphae. The patient was treated by surgical debridement, Amphotericin B and Itraconazol obtaining a complete cicatrization of the wound. S prolificans is a hyaline filamentous fungi, resistant to Amphotericin B and azoles, considered currently an emerging pathogen. It is responsible of localized infections associated to surgery and/or trauma, as well as fatal disseminated infections in immunocompromised hosts, specially oncological, in presence of neutropenia, and transplants. It is important to consider this microorganism in the differential diagnosis of emerging fungal infections.

Key words: Scedosporium prolificans, surgery.

\section{Introducción}

En los últimos años se ha observado un aumento importante de pacientes inmunocomprometidos lo que ha significado un incremento en la incidencia de las infecciones fúngicas oportunis$\operatorname{tas}^{1}$. Las especies clásicas de los géneros Candida, Aspergillus, Cryptococcus y del Orden Mucorales continúan siendo los más frecuentes. Sin embargo, otros hongos de baja virulencia considerados como colonizadores o contaminantes se han agregado a la lista de patógenos en este tipo de pacientes, como: hongos filamentosos hialinos (Fusarium, Paecilomyces, Acremonium, Scedosporium), hongos filamentosos dematiáceos (Bipolaris, Exophiala, Alternaria, Cladophialophora bantiana), hongos levaduriformes de los géneros
Trichosporon, Malassezia, Blastoschizomyces, Rhodotorula, Saccharomyces y otras.

Scedosporium prolificans ha emergido como una causa importante de infecciones localizadas y diseminadas severas, principalmente en individuos inmunocomprometidos ${ }^{2-6}$, neutropénicos ${ }^{7}$, pacientes con enfermedades onco hematológicas ${ }^{8-12}$ y sometidos a trasplantes ${ }^{13,14}$. Se ha comunicado además, un brote nosocomial constituído por 4 casos de infecciones diseminadas fatales en pacientes leucémicos ${ }^{8}$.

Presentamos el primer caso de una infección localizada por $S$. prolificans en Chile.

\section{Caso clínico}

Hombre 27 años, con glomérulo nefritis cró-

Programa de Microbiología, ICBM, Facultad de Medicina, Universidad de Chile (MCDJ).

Laboratorio de Microbiología, Hospital Del Salvador, S.S.M.O. Santiago (CJL).

Servicio de Micología, Centro Nacional de Microbiología, Instituto de Salud Carlos III, Majadahonda, España (AM de la T, JL R-T).

Recibido: 13 enero 2004

Aceptado: 30 enero 2004 
nica y estenosis renal bilateral, en hemodiálisis crónica desde noviembre 1998. En diciembre de 2000 se sometió a transplante renal, en el Hospital Del Salvador de Santiago. El riñón procedía de donante cadáver y fue sometido a isquemia fría durante 8 horas. La cirugía fue prolongada por lesión de la arterial renal. Recibió como tratamiento: prednisona $125 \mathrm{mg}$, azatioprina $150 \mathrm{mg}$, y ciclosporina $1,6 \mathrm{ml}$ cada 12 hrs. Presentó necrosis tubular aguda post-transplante debiendo ser hemodializado. A los 10 días post trasplante fue dado de alta en buenas condiciones.

A los 25 días del trasplante percibió aumento de volumen en la zona operatoria y escurrimiento de secreción por la herida, de ella se obtuvo muestra para estudio microbiológico. El examen directo fue negativo y en el cultivo se aisló un hongo filamentoso. Una semana después se repitió el estudio microbiológico de la secreción, observándose al examen directo hifas hialinas (Figura 1) y en el cultivo se desarrolló el mismo hongo, identificado en base a características macro y micromorfológicas como Scedosporium prolificans. Se practicó ecotomografía en la zona del aumento de volumen fluctuante constatándose una imagen sospechosa de absceso bajo la zona operatoria, por lo cual se internó al paciente, se le practicó aseo quirúrgico más biopsia y se inició tratamiento con anfotericina $B$, según esquema habitual (dosis total propuesta de $1 \mathrm{gr}$ ), manteniéndose la prednisona y ciclosporina. Posteriormente se agregó itraconazol $400 \mathrm{mg}$, el cual se mantuvo durante 2 meses.

El estudio histopatológico realizado con tinciones de hematoxilina eosina, PAS y Gomori Grocott reveló intensa inflamación aguda y crónica, fibrosis y formación de tejido de granula-

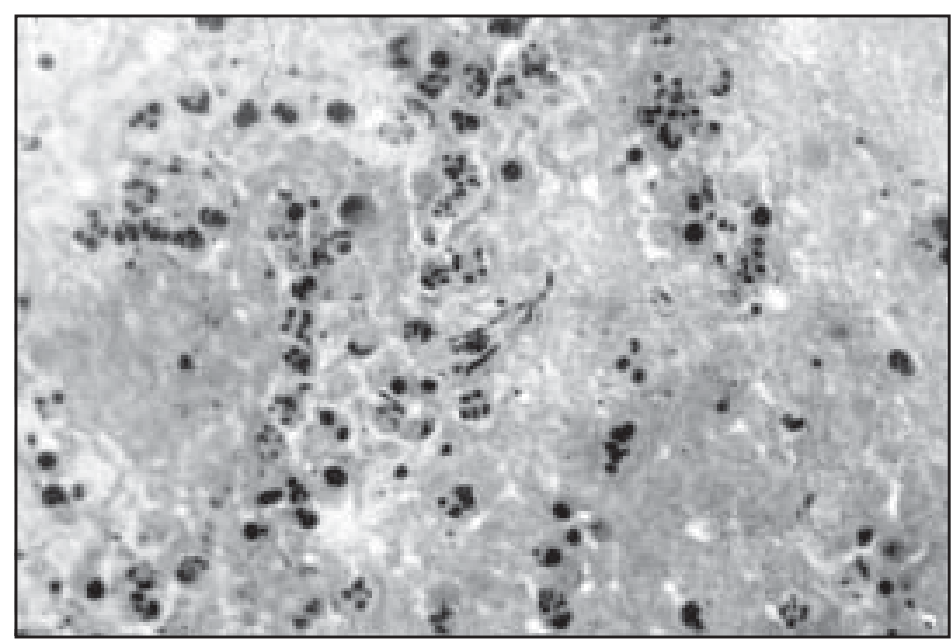

Figura 1. ción, observándose hifas con invasión vascular. La conclusión histopatológica fue de un absceso de pared de naturaleza fúngica. El estudio microbiológico de la biopsia demostró hifas hialinas al examen directo y en el cultivo se desarrolló el mismo hongo aislado previamente.

Al completar $525 \mathrm{mg}$ de anfotericina B presentó deterioro de la función renal (creatininemia se elevó de 1,7 a 3,6 mgrs/dl) suspendiéndose el antifúngico por 3 días, el cual fue reinstalado en forma ambulatoria hasta completar 1 gr. La herida operatoria evolucionó bien retirándose los puntos de sutura a los 10 días. Fue dado de alta a los 16 días de hospitalización en buenas condiciones.

En controles posteriores repetidos a dos años plazo, se comprobó la curación de la infección y cicatrización completa de su herida. Continúa con tratamiento inmunosupresor de ciclosporina $\mathrm{y}$ prednisona.

Se realizó estudio de susceptibilidad in vitro del hongo aislado siguiendo una técnica equivalente a la recomendada por NCCLS. Las CIM obtenidas fueron: anfotericina B $16 \mu \mathrm{g} / \mathrm{ml}$, 5fluorocitosina $128 \mu \mathrm{g} / \mathrm{ml}$, itraconazol $8 \mu \mathrm{g} / \mathrm{ml}$, voriconazol $32 \mu \mathrm{g} / \mathrm{ml}$, y terbinafina $16 \mu \mathrm{g} / \mathrm{ml}$.

La identificación y susceptibilidad a antifúgicos de esta cepa fue confirmada en el Centro Nacional de Microbiología, del Instituto de Salud Carlos III, Majadahonda, Madrid (JL. Rodríguez Tudela y A. Monzón).

\section{Micología}

Características macroscópicas: Colonias de crecimiento rápido (inicia crecimiento a las 72 horas), planas, extendidas, al comienzo blancas, posteriormente llegan a ser grisolivácea a negruzca (Figuras 2 y 3 ).

Características microscópicas: Hifas septadas, hialinas, ramificadas. Conidios unicelulares, hialinos a café-pàlido ovoides a piriforme, 2,5 x 3-13 um en tamaño y de paredes lisas. Se originan en pequeños grupos de anélides en forma de frasco, que se disponen solitariamente o en racimos a lo largo de la hifa vegetativa (Figura 4).

Características fisiológicas: No asimila sacarosa, ribitol, xilitol, eritritol y l-arabinitol, sensible a cicloheximida, asimila almidón, ureasa positiva, licuación de gelatina 


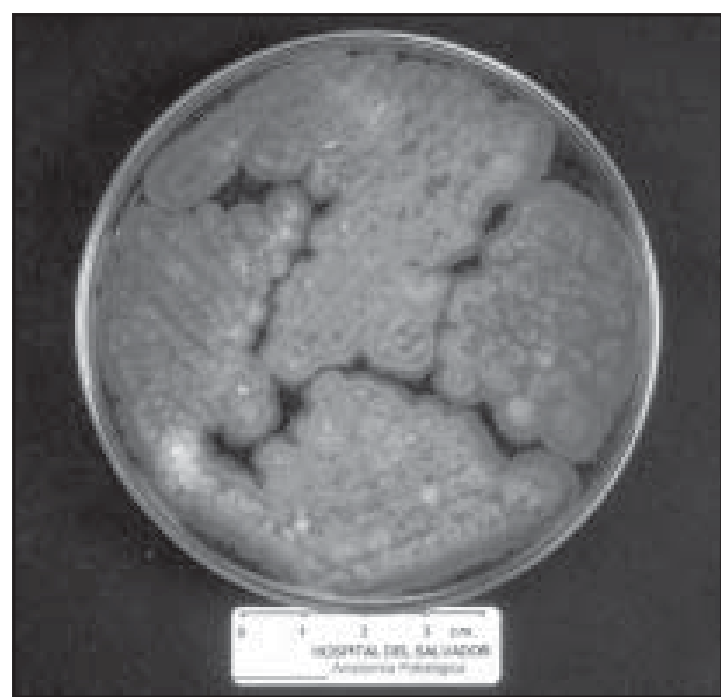

Figura 2.

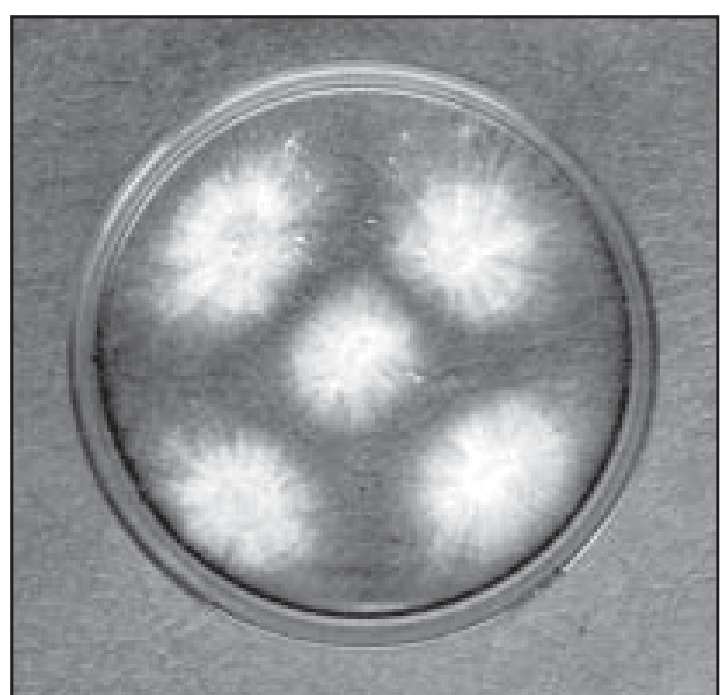

Figura 3.

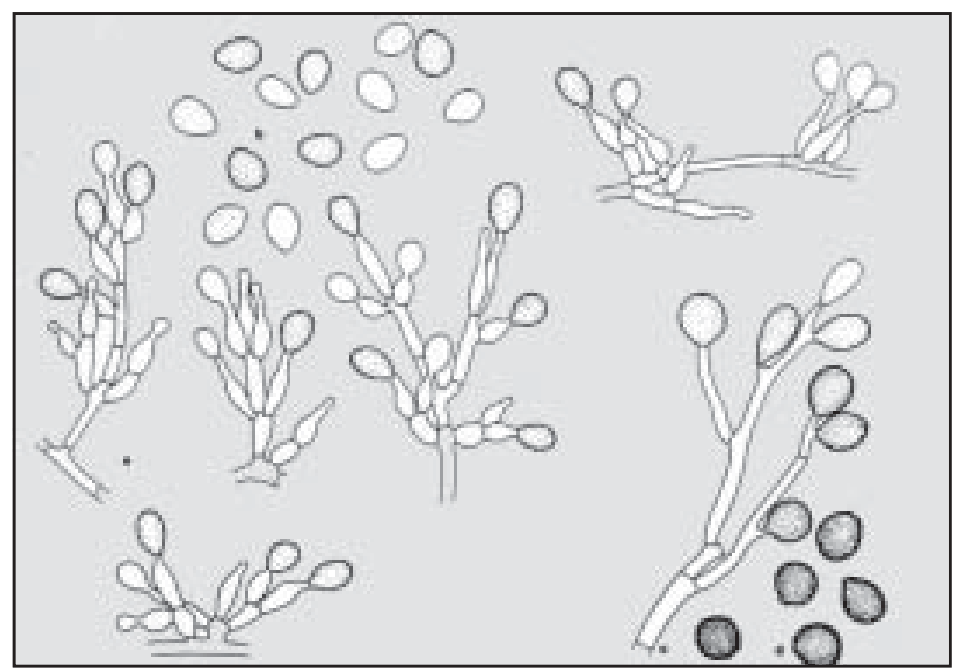

Figura 4. Morfología de Scedosporium prolificans.(Reproducido de $\operatorname{ref}^{25}$ ).
Reino: Hongo; División: Ascomycota; Clase: Euascomycetes; Orden: Microascales; Familia: Microascaceae; Género: Scedosporium. Este género tiene dos especies importantes como patógenos humanos, Scedosporium apiospermum - estado asexual de Pseudallescheria boydii- y Scedosporium prolificans.

El hábitat natural de $S$. prolificans es el suelo ${ }^{3,14}$, pero también ha sido aislado de tierra de maceteros y en el aire de una habitación de un paciente leucémico ${ }^{15} \mathrm{y}$ considerado como un patógeno emergente en ciertas áreas del mundo como España y Australia ${ }^{3,5,14,15}$. El reservorio probable en nuestro paciente, según planteamiento del equipo positiva y crece $37^{\circ} \mathrm{C}$. Es resistente a anfotericina $\mathrm{y}$ azoles in vitro.

\section{Discusión}

En 1974, Hennebert \& Desai aislaron en el suelo de Bélgica un hongo denominado Lomentospora prolificans. Malloch y Salkin (1984) describieron un hongo dematiáceo, Scedosporium inflatum como un nuevo patógeno, aislado de un niño con osteomielitis. Gueho y de Hoog (1991) como resultado de estudios taxonómicos encontraron $100 \%$ de homología de ADN entre estas dos especies y la denominaron Scedosporium prolificans.

La clasificación taxonómica corresponde a: tratante, habría sido el aire ambiental, y vía de infección, la caída de conidios sobre la herida abierta durante el acto quirúrgico - no se dispone de flujo laminar en los pabellones quirúrgicos del establecimiento-, el que fuera laborioso y más prolongado de lo habitual por complicaciones durante la sutura de los vasos sanguíneos.

El espectro de infección es amplio ${ }^{2,3}$ causando colonizaciones transitorias asintomáticas, infecciones localizadas de piel ${ }^{17}$, tejidos blandos, conducto auditivo externo, osteomielitis, artritis, queratitis ${ }^{21}$, sinusitis de origen denta ${ }^{19}$, tracto respiratorio e infecciones diseminadas, a menudo falitis $^{18}$, y fungemias asociadas a trauma, cirugía fatales $2,8,10,13$ como endocarditis, meningoence- 
e inmunodepresión: $\operatorname{SIDA}^{4,6,16}$, cánceres hematológicos y trasplantes de órganos ${ }^{3,12}$. Ciertas técnicas invasoras, implantes protésicos y tratamiento inmunosupresor también contribuyen al aumento de estas micosis.

Scedosporium prolificans. Es un hongo filamentoso con resistencia in vitro a todos los antifúngicos en uso actualmente, por lo cual el manejo terapéutico puede llegar a ser difícil y no está claro aún cuál es la terapia antifúngica ideal. A pesar de que este hongo muestra resistencia in vitro a los antifúngicos, algunos casos tratados han evolucionado favorablemente ${ }^{7,20,22}$. No obstante de que existe discordancia entre los resultados de estudios in vitro y respuesta clínica, hecho descrito por lo demás para otras infecciones fúngicas, creemos que los ensayos de susceptibilidad tienen un rol orientador siendo de utilidad de ejecución. Los nuevos antifúngicos azólicos como voriconazol, posaconazol y ravuconazol han demostrado tener una excelente eficacia ${ }^{23,24}$.

La cirugía en las infecciones localizadas está absolutamente indicada, lo mismo que la eliminación de la inmunodepresión y suspensión disminución de los agentes inmunosupresores mientras dure la terapia de esta infección. En nuestro paciente creemos que el aseo quirúrgico tuvo mayor relevancia que la terapia antifúngica en la curación del absceso; sin embargo, no podemos descartar que los antifúngicos tuvieron un efecto benéfico también.

En conclusión, la prolongación de la vida de pacientes inmunocomprometidos, los procedimientos invasores a los cuales son sometidos, el amplio uso de agentes inmunosupresores y la quimioterapia citotóxica agresiva juegan un rol importante en la emergencia de nuevos patógenos fúngicos.

Scedosporium prolificans debe ser considerado en el diagnóstico diferencial de las infecciones fúngicas localizadas y diseminadas en las situaciones clínicas anteriormente mencionadas y agregarse a la lista de los hongos filamentosos aislados en nuestro país, después de Aspergillus y Fusarium.

Con respecto al tratamiento, en la mayoría de las infecciones diseminadas es necesario la eliminación de la inmunodepresión para obtener algún tipo de respuesta ${ }^{7}$. En infecciones localizadas, como en este caso, es importante la cirugía unida al tratamiento antifúngico.

\section{Resumen}

Se presenta el primer caso de infección por Scedosporium prolificans en nuestro país. Se trata de un paciente de 27 años, sexo masculino, con insuficiencia renal crónica e hipertensión, sometido a trasplante renal, en tratamiento con ciclosporina, azatioprina y prednisona. A los 25 días posttransplante presentó un aumento de volumen fluctuante bajo la herida operatoria. En tres muestras de exudado purulento y tejido obtenido finalmente por cirugía resolutiva se aisló $S$. prolificans como agente único. El estudio histológico reveló estructuras compatibles con hifas septadas. El paciente se trató con aseo quirúgico, anfotericina $\mathrm{B}$ e itraconazol lográndose la curación y cicatrización completa de la herida. S prolificans es un hongo filamentoso hialino resistente a anfotericina B y azoles, considerado actualmente un patógeno emergente, responsable de infecciones localizadas asociadas a cirugía y/o trauma, como también de infecciones diseminadas fatales en hospederos inmunocomprometidos especialmente pacientes neutropénicos, oncológicos y trasplantados. Es importante considerar este hongo en el diagnóstico diferencial de infecciones fúngicas emergentes.

\section{Bibliografía}

1.- Ponton J, Ruchel R, Clemons K V et al. Emerging pathogens. Med Mycol 2000; 38 Suppl 1: 225-36.

2.- Berenguer J, Rodríguez-Tudela J L, Richard C et al. Deep infections caused by Scedosporium prolificans: A report on 16 cases in Spain and a review of the literature. Scedosporium prolificans Spanish study Group. Medicine (Baltimore) 1997; 76: 256-65.

3.- Gosbell I, Morris M, Gallo J et al. Clinical, pathologic and epidemiologic features of infection with Scedosporium prolificans: four cases and review. Clin Microbiol Infect 1999; 5: 672-86.

4.- De Pablos M, Aldamiza M, Portu J J, Soto M J, Michaus L, Lezaun M J, Jimenez J C. Scedosporium prolificans infection in an AIDS patient. Int Conf AIDS 1996; 7-12; 11 (1): 94 (abstract $N^{\circ}$ MoB 1230)

5.- Idigoras P, Pérez-Trallero E, Pineiro L, Larruskain J, Lopez-Lopategui M C, Rodríguez N, González J M. Disseminated infection and colonization by Scedosporium prolificans: a review of 18 cases, 19901999. Clin Infect Dis 2001; 32 (11): E158-65.

6.- Nenoff P, Gutz U, Tintelnot K, Bosse-Henck A, Mierzwa M, Hofmann J, Horn LC, Haustein F. Disseminated mycosis due to Scedosporium prolificans in an AIDS patient with Burkitt lymphoma. Mycoses 1996; 39: 461-5.

7.- Bouza E, Muñoz P, Vega L, Rodríguez-Crèixems M, Berenguer J, Escudero A. Clinical resolution of Scedosporium prolificans fungemia associated with reversal of neutropenia following administration of granulocyte colony-stimulating factor. Clin Infect Dis 1996; 23: 192-3.

8.- Alvarez M, López Ponga B, Rayon C, García Gala J, Roson Porto M C, González M, Martínez-Suárez J, Rodríguez-Tudela J L. Nosocomial outbreak by Scedosporium prolificans (inflatum): four fatal cases in leukemic patients. J Clin Microbiol 1995; 33: 3290-5.

9.- De Batlle J, Motjè M, Balanza R, Guardia R, Ortiz R. Disseminated infection caused by Scedosporium 
prolificans in a patient with acute multilineal leukemia. J Clin Microbiol 2000; 38: 1694-5.

10.- Maertens J, Lagrou K, Deweerdt H, Surmont I, Verhoef GE, Verhaegen J, Boogaerts M A. Disseminated infection by Scedosporium prolificans: an emerging fatality among haematology patients. Case report and review. Ann Hemathol 2000; 79: 340-4.

11.- Westerman D A, Speed B R, Prince H M. Fatal disseminated by Scedosporium prolificans during induction therapy for acute leukemia: a case report and literature review. Pathology 1999; 31: 393-4.

12.- Spielberger $\mathrm{R} \mathrm{T}$, Tegtmeier $\mathrm{B}$, O’Donnell $\mathrm{M}$, Ito J. Fatal Scedosporium prolificans ( $S$ inflatum) fungemia following allogeneic bone marrow transplantation: report of a case in the United States. Clin Infect Dis 1995; 21: 1067.

13.- Rabodonirina M, Paulus S, Thevenet $\mathrm{F}$ et al. Disseminated Scedosporium prolificans (S inflatum). Infection after single-lung transplantation. Clin Infect Dis 1994; 19: 138-42.

14.- López L, Gastelurrutia L, Cuenca-Estrella M, Monzón A, Barròn J, J Hernández J L, Pérez R. Infección y colonización por Scedosporium prolificans. Enfermedades Infecciosas y Microbiologia Clìnica 2001; 19: 308-13.

15.- Idigoras P, García-Arenzana J M, Saenz J R, Pineiro L, Marin J. Isolation of Scedosporium prolificans on the air in the room of a patient with leukemia and disseminated infection with this fungus. Enferm Infecc Microbiol Clin 2000; 18 : 426-7.

16.- Hopwood V, Evans E G, Matthews J, Denning D W. Scedosporium prolificans, a multi-resistant fungus, from a U.K AIDS patient. J Infect 1995; 30: 153-5.

17.- Gillum P S, Gurswami A, Taira J W. Localized cutaneous infection by Scedosporium prolificans (inflatum) Int J Dermatol 1997; 36: 297-9.

18.- Madrigal V, Alonso J, Bureo E, Figols F J, Salesa R. Fatal meningoencephalitis caused by Scedosporium inflatum (S. prolificans) in a child with lymphoblastic leukemia. Eur J Clin Microbiol Infect Dis 1995; 14: 601-3.

19.- Chikhani L, Dupont B, Guilbert F, Improvisi L, Corre A, Bertrand J C. Uncommon fungal maxillary sinusitis of dental origin due to Scedosporium prolificans. Rev Stomatol Chir Maxillofac 1995; 96: 66-9.

20.- Barbaric D, Shaw P J. Scedosporium infection in immunocompromised patients: successful use of liposomal amphotericin B and Itraconazole. Med Pediatr Oncol 2001; 37: 122-25.

21.- Sullivan L J, Snibson G, Joseph C, Taylor H R. Scedosporium prolificans sclerokeratitis. Aust N Z J Ophthalmol 1994; 22: 307-9.

22.- Pickles R W, Pacey D E, Muir D B, Merrell W H. Experience with infection by Scedosporium prolificans including apparent cure with fluconazole therapy. J Infect 1996; 33: 193-7.

23.- Cuenca Estrella M, Ruiz Diez B, Martínez Suárez JV, Monzon A, Rodríguez Tudela J L. Comparative in vitro activity of voriconazole (UK-109,496 ) and six other antifungal agents against clinical isolates of Scedosporiumn prolificans and Scedosporium apiospermiun. J Antimicrob Chemother 1999; 43: 14951.

24.- Meletiadis J, Mouton J W, Rodríguez Tudela J L, Meis J F, Verweii P E. In vitro interaction of terbinafine with itraconazole against clinical isolates of Scedosporiom prolificans. Antimicrob Agents Chemother 2000; 44: 470-2.

25.- De Hoog G S, Guarro J, Gené J L, Figueras M J. Atlas of Clinical Fungi. $2^{\text {nd }}$ Ed. 2000.

Correspondencia a:

M. Cristina Díaz J

E-mail:mcdiaz@med.uchile.cl 